\title{
Aligning systemic infrastructure decisions with social outcomes
}

\author{
In the second article from the Institution of Civil Engineers' thought leadership programme, Tom \\ Dolan and Ellie Cosgrave from University College London propose a new way to ensure that \\ infrastructure investment delivers the desired benefits for society.
}

The UK Council for Science and Technology (CST) concluded 7 years ago that, 'We do not believe national infrastructure can continue on its current trajectory' (CST, 2009). It said delivery and governance were 'highly fragmented' and resilience against systemic failure was 'significantly weakening'.

The UK government responded by creating Infrastructure UK - now the Infrastructure and Projects Authority (IPA), publishing annual national infrastructure plans and, from this year, a 5-year national infrastructure delivery plan (IPA, 2016a).

It also developed and regularly updates an infrastructure pipeline of over 600 projects and programmes costing $\mathrm{f} 425$ billion (IPA, 2016b), and launched a National Infrastructure Commission and a consultation on strategic national infrastructure assessment (Dolan, 2015; HMG, 2016).

\section{Social outcomes missing}

But while the approaches yield many potential benefits, we believe they are not yet sufficient to address the CST's warning. The plans, delivery plans and assessments are at risk of failing to capture the opportunity for infrastructure to enable the broad range of social outcomes we know infrastructure can support.

For example, schools, healthcare facilities and other social spaces are considered outside the scope of infrastructure planning, and look set to be excluded from the scope of the national infrastructure assessment (HMG, 2016).

If our infrastructure planning system artificially separates economic and social outcomes - and the requisite investment strategies - we constrain our ability to develop infrastructure capable of enabling the social outcomes expected.

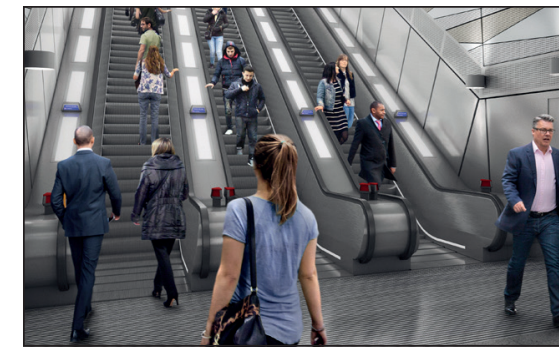

The proposed new toolkit will deliver a clearer vision of the social benefits of future infrastructure projects at a system level

\section{Systemic challenge}

The need to take a whole-system approach has been recognised in government plans, but what this means in practice is less clear. System problems are shared problems: they are caused by no one party in isolation, and can be solved by no one party in isolation.

System problems emerge as a consequence of interaction between system components - including the political, social and economic context in which they are embedded - and are best managed collaboratively.

As part of its thought leadership programme, the Institution of Civil Engineers therefore proposes developing a systemic toolkit comprising a set of transparent, systemic, structured, interconnected and flexible methodologies. They would cover systemic infrastructure visioning, performance and needs analysis, and option identification and selection.

\section{Proposed methodologies}

Our methodology will facilitate vision development. We will also develop a methodology to identify infrastructure system 'performance gaps'. This will help identify infrastructure needs at the system rather than sector level, and frame need in terms independent of possible options to address the need.

A systematic methodology is then needed to identify possible options for improving infrastructure system performance. Finally, selection criteria to evaluate possible options should link to the established vision, ensuring that infrastructure decisions contribute positively to, or have no detrimental impact on the ability to manage system problems.

\section{Benefits and opportunities}

The proposed toolkit, if developed and implemented collaboratively, can support systemic decision making.

Potential benefits may include improved capability to manage the risk of system problems, opportunities to innovate in response to needs framed at the system rather than sector level, and societal benefits from explicitly aligning infrastructure decision making with social outcomes.

\section{References}

CST (Council for Science and Technology) (2009) A National Infrastructure for the 21st Century. Council for Science and Technology, London, UK.

Dolan T (2015) Infrastructure Commission: what are the opportunities and how should it work? Infrastructure Intelligence, 27 October. See http://www.infrastructure-intelligence.com/ article/oct-2015/infrastructure-commissionwhat-are-opportunities-and-how-should-it-work (accessed 06/09/2016).

HMG (Her Majesty's Government) (2016) https:// www.gov.uk/government/organisations/ national-infrastructure-commission (accessed 26/08/2016).

IPA (Infrastructure and Projects Authority) (2016a) National Infrastructure Delivery Plan 2016 to 2021 (No. PU1901). HM Treasury, Infrastructure and Projects Authority, London, UK.

IPA (2016b) National Infrastructure Pipeline

2016. HM Treasury, Infrastructure and Projects Authority, London, UK. See https://www.gov.uk/ government/publications/national-infrastructurepipeline-2016 (accessed 26/08/2016) 